\title{
Dual-Band Pin-Patch Antenna for Wi-Fi Applications
}

\section{Peter Callaghan and John C. Batchelor, Senior Member, IEEE}

This is an accepted pre-published version of this paper.

(C) 2008 IEEE. Personal use of this material is permitted. Permission from IEEE must be obtained for all other uses, in any current or future media, including reprinting/republishing this material for advertising or promotional purposes, creating new collective works, for resale or redistribution to servers or lists, or reuse of any copyrighted component of this work in other works.

The link to this paper on IEEE Xplore ${ }^{\circledR}$ is

http://dx.doi.org/10.1109/LAWP.2008.2004885

The DOI is: 10.1109/LAWP.2008.2004885 


\title{
Dual-Band Pin-Patch Antenna for Wi-Fi Applications
}

\author{
Peter Callaghan and John C. Batchelor, Senior Member, IEEE
}

\begin{abstract}
The pin-patch antenna provides a low-profile monopolar element suitable for wireless access point requirements. This design concept is extended here to provide dual-band operation. Experimental results are compared to predictions from a FDTD package confirming monopolar radiation in two bands at $2.4 \mathrm{GHz}$ and $5.7 \mathrm{GHz}$. This band spacing can be adjusted from 1.8:1 to more than $4: 1$.
\end{abstract}

Index Terms - multiband antennas, pin loaded patches, Radio over fibre, remote antenna units

\section{INTRODUCTION}

$\mathrm{R}$ ECENT years have seen the increased use of wireless data systems with the expansion of WLAN/WiFi services. Based on IEEE $802.11 \mathrm{~b} \& \mathrm{~g}$ standards a number of operators are offering PCN (Personal Communications Network) with wireless connectivity at $2.4 \mathrm{GHz}$. The links are for limited range, usually less then $10 \mathrm{~m}$, with the target application being for in-building communications. Whilst a vertical monopole antenna can provide an adequate solution having an antenna height of $30 \mathrm{~mm}$ [1] there are occasions where a lower profile element would be desirable. One example would be for a multiband ceiling mount access point [2]. To reduce the height there are a number of options the antenna designer can consider. PIFA-type antennas can be considered but these can suffer from asymmetry in their radiation patterns. For the lowest profile a circular patch antenna can provide a monopolar type radiation pattern when operated in the higher order TM01 mode [3]. However this can have a relatively large footprint (an air-spaced disc would have a diameter of $145 \mathrm{~mm}$ ). As an alternative pin-loaded plate antenna structures have been reported. Initial designs considered a square plate $[4,5]$ but in $[6]$ a circular patch was demonstrated. This was extended to provide increased bandwidth by use of a capacitive feeding element in [7]. The height $(<\lambda / 10)$ of the pin-patch antenna can therefore be similar to a TM01 Patch antenna but with half the diameter, providing the most compact solution.

Whilst the reported pin-patch antenna designs offer a suitable solution for a single band wireless access point, IEEE 802.11a also allows for connectivity in the 5 GHz ISM bands - and with IEEE802.11n pending pressure is on to provide interoperability between both bands. Similarly for mobile communication applications a pin-patch antenna would need to provide a dual-band performance in the 900 and $1800 / 2000 \mathrm{MHz}$ bands. Low-profile dual-band antenna solutions based upon modified PIFAs have been demonstrated for roof-mount automotive cellular applications [8], but these suffer from pattern asymmetry. In this letter we describe how the basic pin-patch antenna can be modified to provide operation in two bands with spacings of $\sim 2: 1$ to meet these dual-band requirements and maintain the desired omni-directional azimuth pattern. 

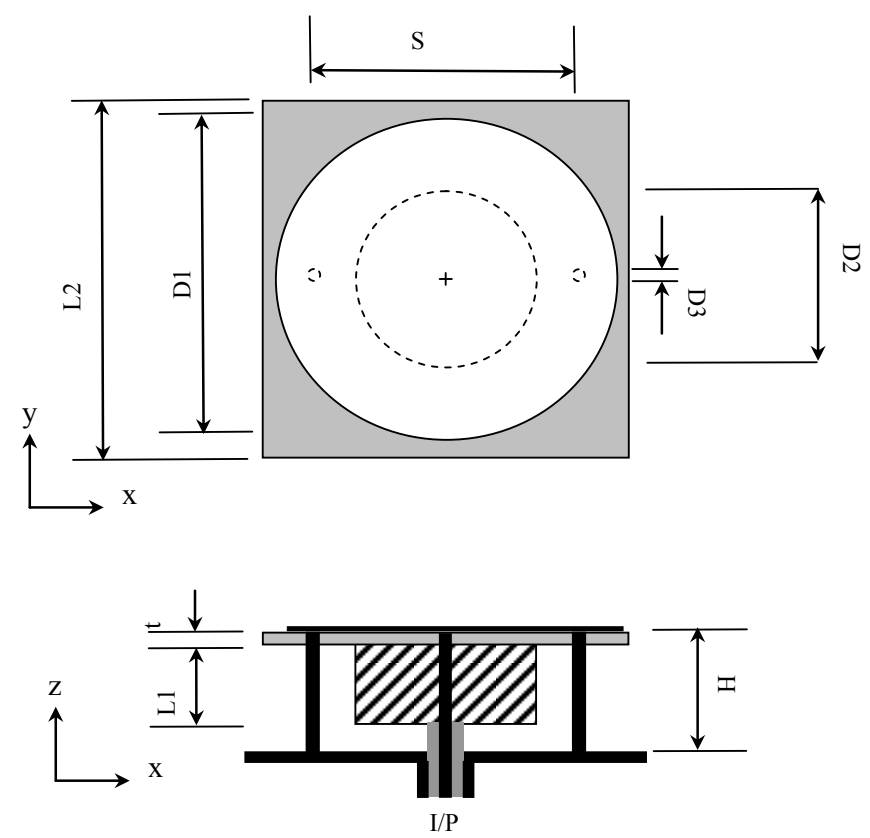

Fig. 1. Dual-Band Pin-Patch Antenna Design. D1 $=28.0 \mathrm{~mm}, \mathrm{D} 2=14.8 \mathrm{~mm}$, $\mathrm{D} 3=1 \mathrm{~mm}, \mathrm{H}=10.0 \mathrm{~mm}, \mathrm{~S}=18.2 \mathrm{~mm}, \mathrm{~L} 1=7.1 \mathrm{~mm}, \mathrm{~L} 2=32 \mathrm{~mm}, \mathrm{t}=0.4 \mathrm{~mm}$.

\section{ANTENNA STRUCTURE}

The basic antenna structure can be seen in Fig. 1. It is essentially a thick circular patch antenna but with two additional grounding pins. The fundamental mode of resonance of a circular patch is the TM11 mode that radiates normal to the patch. This occurs when the patch diameter is approximately $\lambda / 3.4(37 \mathrm{~mm}$ for air-spaced patch at $2.4 \mathrm{GHz})$. Introducing the shorting pins, and feeding centrally effectively suppresses this mode but allows excitation of the higher TM01 mode. Usually the TM01 mode occurs when the patch diameter is approximately $\lambda / 1.6(76 \mathrm{~mm}$ for air-spaced patch at $2.4 \mathrm{GHz})$ but the shorting pins cause a TM01 resonance at a much smaller diameter $(<30 \mathrm{~mm})$. The TM01 mode radiates in the plane of the patch providing the monopolar pattern desirable for wireless communications applications. The element may also be thought of as a top-loaded monopole with the shorting pins providing matching at a lower operating frequency. In $[5,6]$ it was shown that the resonant frequency is determined primarily by the height, $\mathrm{H}$, and diameter, D1, of the patch but also the diameter, D2, of the central feed post and the spacing, S, of the grounding pins. In [2] it was also shown that the bandwidth and impedance also varies with resonant frequency when the patch diameter was varied for a fixed height and pin geometry. Adjusting these parameters produces a single band of operation, with the next higher order mode nearly an order of magnitude higher. For single band operation this is ideal.

It was noted from an analysis of this element using commercial FDTD software that the monopolar radiation property is actually wideband but the antenna is only matched over a narrow band. This can be understood as the natural frequency of the TM01 is nearly 3 times higher than the forced resonant frequency and also because the lower order modes are suppressed. To create a dual-band pin-patch design a method of matching at a higher frequency was sought such that the fundamental mode of excitation remains unchanged.

TABLE I

VAlues of Bush Diameter to MATCh SeCOND BAND FOR DifFERENT BUSH LENGTHS

\begin{tabular}{ccc}
\hline \hline Length L $(\mathrm{mm})$ & Diameter D2 $(\mathrm{mm})$ & $\mathrm{D} 2 /(\mathrm{S}-\mathrm{D} 3) \%$ \\
\hline 5 & 10.4 & 60 \\
6 & 12.2 & 71 \\
7.1 & 14.8 & 86 \\
8 & 16.6 & 96
\end{tabular}

In our design the feeding structure used a standard flange mount SMA connector. This has a centre pin of diameter $1.3 \mathrm{~mm}$ with a $2.5 \mathrm{~mm}$ PTFE dielectric. To construct the antenna of Fig. 1 the SMA pin is inserted inside a larger metallic bush to achieve a larger feed post diameter. If the length of this bush, L1, is reduced a step in the centre post diameter is formed. The PTFE dielectric of the SMA connector can be extended to support this conductive bush. This structure was modelled using a commercial FDTD software package and this showed that introducing the step in the centre post diameter could introduce a 
second resonance, providing dual-band operation. The stepped post appears to provide an L-C types series matching arrangement to the TM01 current mode of excitation, such that the primary characteristics of the element remained unchanged.

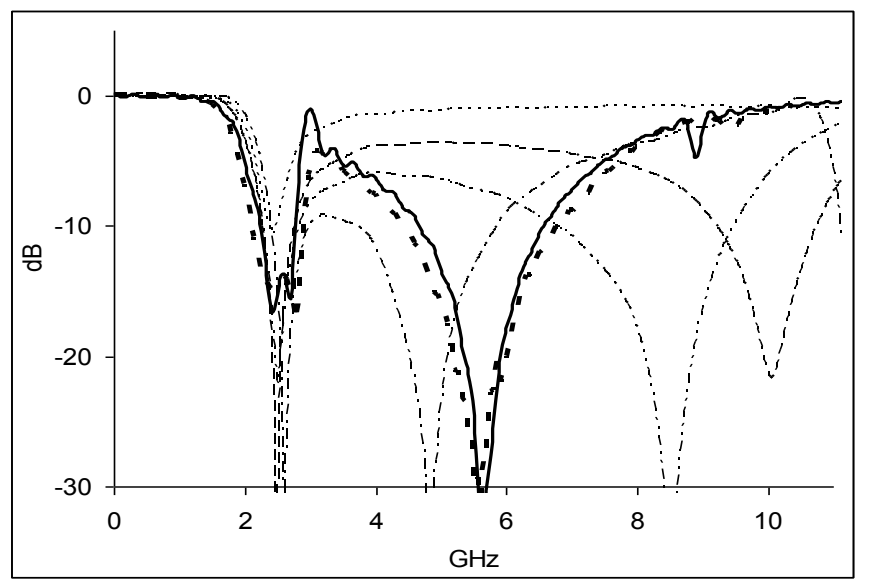

Fig. 2. Return loss of Antenna of Fig.1 for different centre post step lengths (L1).

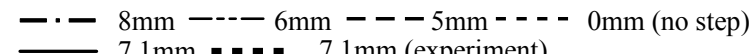

To illustrate the dual-band characteristics of this structure return loss plots for different length bushes are plotted in Fig. 2. As can be seen the fundamental resonance around $2.5 \mathrm{GHz}$ remains virtually unchanged while the second higher frequency resonance is directly affected by the bush length, L1. Here the bush diameter has been adjusted to achieve a match at each length, with the bush diameter increasing for an increase in length. The bush diameter required to provide a match for different bush lengths are given in Table 1. The third row in Table 1 expresses the diameter of the step, D2, as a percentage of the gap between the pins (S-D3). Note, for a length of $8 \mathrm{~mm}$ the diameter of the bush is at a maximum as it is nearly touching the shorting posts (98\% of available gap). This limit of an $8 \mathrm{~mm}$ length represents the lowest band spacing of around 1.8:1. However reducing the bush length increases the band spacing considerably - with a 4:1 band spacing seen in Fig. 2. In the limit with a bush length of zero the structure degenerates to the single resonance antenna of [4]. Of interest at the longer bush lengths the two resonances begin to combine to provide a 'single' band of operation but having a wide bandwidth. For L1 $=8 \mathrm{~mm}$ the return loss is (almost) less than $-10 \mathrm{~dB}$ from $2.4 \mathrm{GHz}$ to $5.9 \mathrm{GHz}$. With further optimisation this approach may provide a wide bandwidth single band antenna.

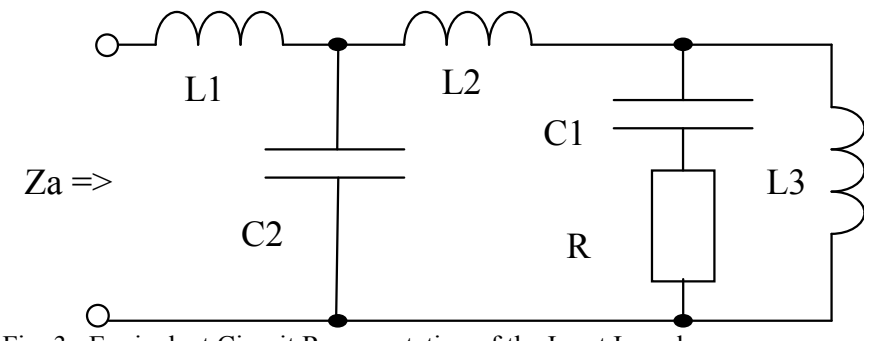

Fig. 3. Equivalent Circuit Representation of the Input Impedance.

The ability to tune the higher band whilst keeping the fundamental resonance fixed by modifying only the dimensions of the step may be explained using an equivalent circuit representation for the input impedance of the antenna ( $\mathrm{Za}$ ). We present an outline model in Fig. 3. For a basic plate antenna without any shorting pins the antenna is essentially a top-load dipole and the equivalent circuit is a series resonance circuit. This is constituted by the elements L1+L2, C2 and R, where R is less than $50 \mathrm{Ohm}$. Adding the shorting pins introduces the shunt inductance L3 providing a transforming network with (L1+L2) to match the input to $50 \mathrm{Ohm}$. The inductance $\mathrm{L} 1+\mathrm{L} 2$ includes the inductance of the centre feed pin and when a stepped centre pin is used an additional capacitance, $\mathrm{C} 2$, is introduced that dissects the inductance into the 2 separate elements, L1 and L2. This additional capacitance excites the second, higher frequency resonance. 

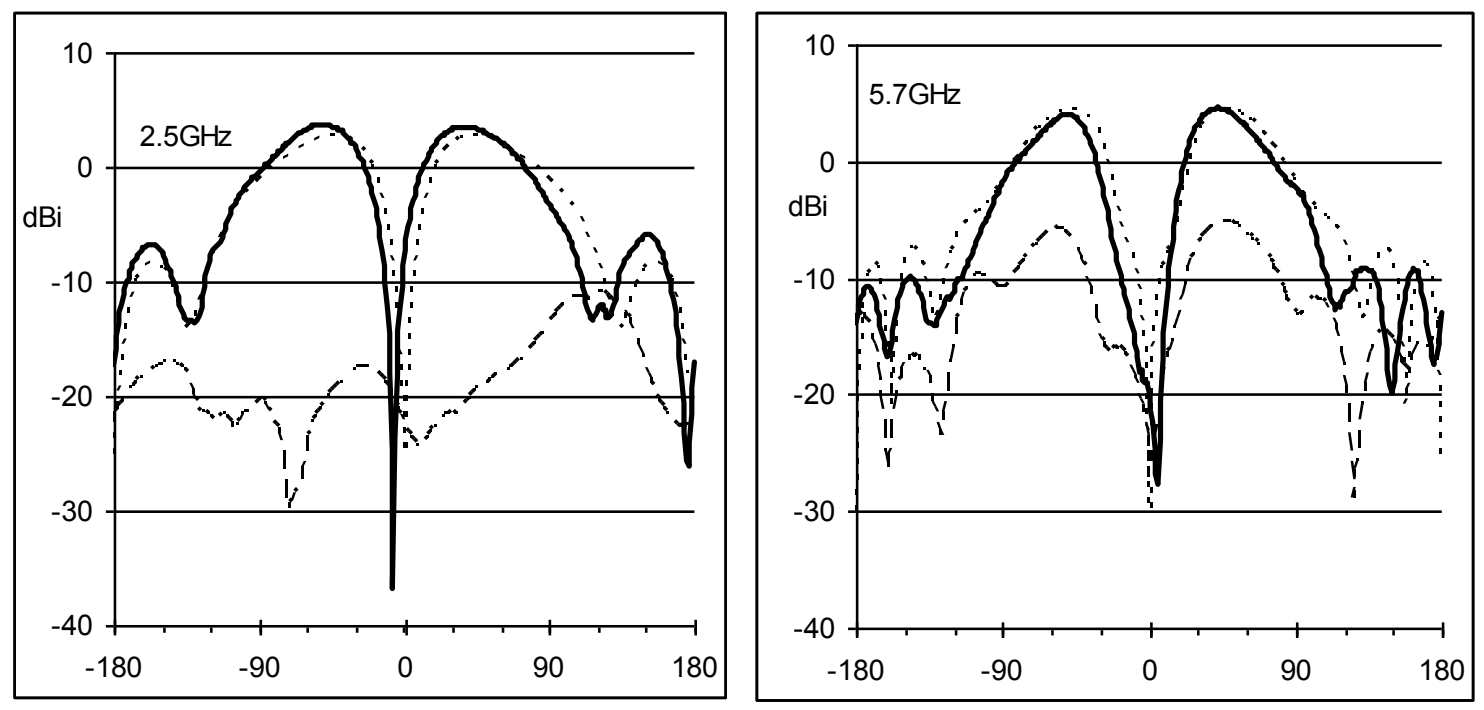

(a) Elevation (y-z plane)
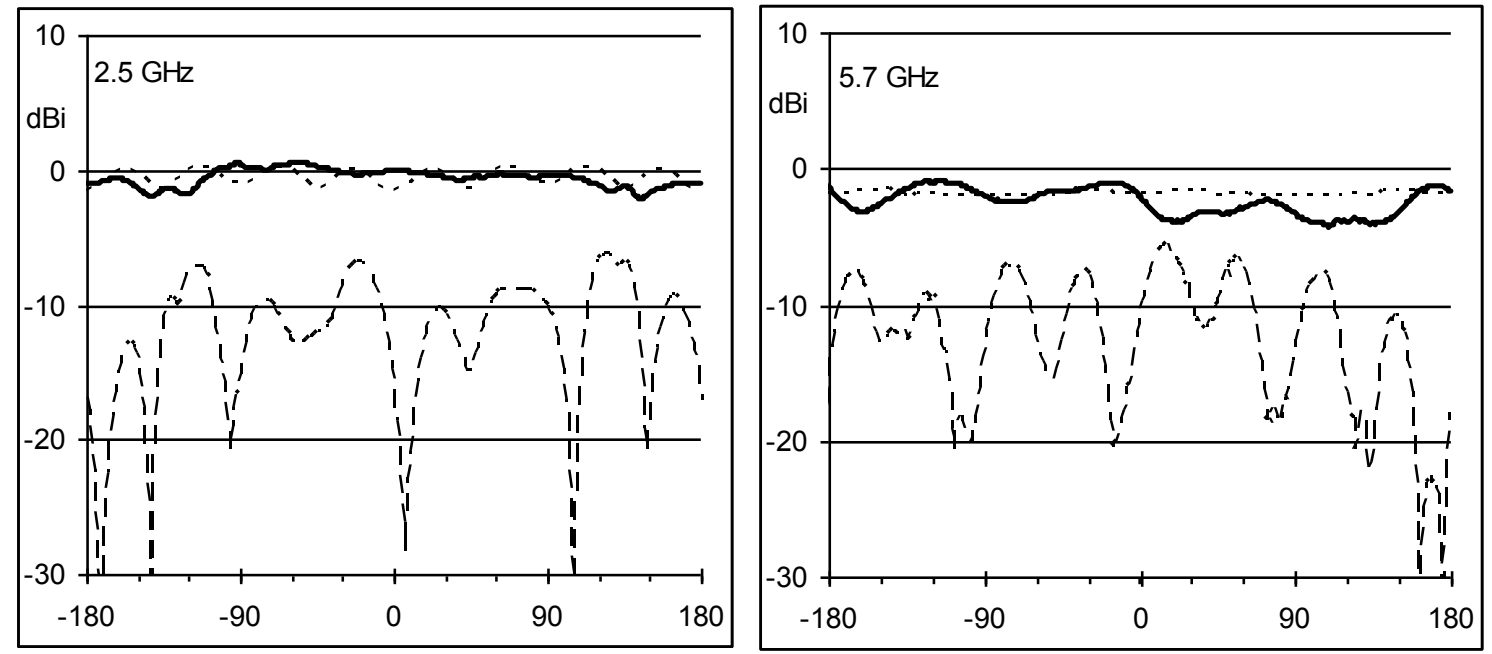

(b) Elevation (x-y plane)

Fig. 4. Radiation Patterns.

\section{- Co-polar - - - - Crosspolar …...... - -Co-polar (Simulated)}

As the position along the centre increase L2 increases, L1 decreases and the frequency of this second resonance falls. To maintain a good match the value of $\mathrm{C} 2$ needs also to increase, and the diameter of the step increases. As C2 increases the value of $\mathrm{C} 1$ falls as the two capacitances are sharing the top plate area and so $(\mathrm{C} 1+\mathrm{C} 2)$ is, to a first approximation, constant. Similarly while the individual values of L1 and L2 may vary their sum remains constant. The fundamental resonance is dependant upon $(\mathrm{C} 1+\mathrm{C} 2)$ and $(\mathrm{L} 1+\mathrm{L} 2)$ and therefore remains fixed. 


\section{EXPERIMENTAL RESULTS}

To confirm the dual-band tuning concept, an experimental design according to Fig. 1 was built and tested. The top circular plate was etched onto an FR4 substrate of $0.4 \mathrm{~mm}$ thickness (t) allowing the pins to be soldered for prototyping. This does introduce a small dielectric layer between the top plate the top of the centre post. However these areas are shorted together so this has minimal effect on the basic design. The experimental design has a patch diameter of $\sim \lambda / 4.5$ at the lower band of operation, making it nearly three times smaller than an equivalent TM01 patch antenna. The height of the antenna is $\sim \lambda / 12$ but is sufficient to allow tuning of a second band as discussed above.

The measured input impedance of the experimental design is compared to predictions from the FDTD package in Fig. 2 with good agreement. The lower band is centred at $2.5 \mathrm{GHz}$ with a $22 \%$ bandwidth (-10dB return loss points). The higher band is centred at $5.7 \mathrm{GHz}$ with a $34 \%$ bandwidth (UK ISM bands). This provides more than sufficient bandwidth for dual-band access point operation. Similarly measured radiation patterns are compared to predictions in Fig. 4 for the three principal planes at the band centres (2.5 GHz for the lower and $5.7 \mathrm{GHz}$ for the upper, both being the ISM bands). The antenna provides nominally monopolar radiation across each band, with near omni-directional patterns in the azimuth plane for vertical polarisation. Being a monopole on a finite ground plane ( $150 \mathrm{~mm}$ square) the peak of radiation is directed at $\sim 30^{\circ}$ elevation angle, as shown by both model and experiment. The elevation cuts give good agreement against the predictions. Small discrepancies are seen in the azimuth patterns which are attributed to measurement error. The azimuth pattern was taken at $90^{\circ}$ elevation where the antenna gain is varying, hence small measurement variations can give rise to $1-2 \mathrm{~dB}$ error.

\section{CONCLUSIONS}

It has been shown that the pin-patch antenna can provide two bands of operation by introducing a step in the centre post. The lower resonant frequency is determined by the overall patch dimensions and the higher resonance is then controlled by varying only the centre post dimensions, providing ease of design. The width of each band of an experimental design was shown to be more than $20 \%$ - although it was noted that close band spacings can merge to form a single large band of operation. Good agreement was found between experiment and theory for both impedance and radiation pattern. Having a height of less than $\lambda / 10$ and a foot print 3 times smaller than an equivalent patch antenna makes this an ideal candidate for wireless access points such as indoor ceiling-mount applications.

\section{ACKNOWLEDGMENT}

We would like to thank Simon Jakes for help in constructing the antennas.

\section{REFERENCES}

[1] Y-W. Jang and H-C Go: 'A low-profile broadband 16-mm diameter post-type monopole antenna ceiling-mounted in a building', Micro. and Opt. Tech. Lett., Vol. 41, No. 5, 5 June 2004, pp 395-397.

[2] P. Callaghan and J. C. Batchelor: 'Multiband Low-Profile Antenna for Remote Antenna Unit Pico Cell Applications', IEEE Trans. Ant \& Propag. Accepted for publication.

[3] L. Economou and R.J.Langley: 'Patch antenna equivalent to simple monopole', IEE Elect. Letts, 24 April 1997, Vol. 33, pp 727-729.

[4] Ch. Delaveuad, Ph. Leveque and, B. Jecko: 'New kind of Microstrip Antenna: the Monopolar Wire-Patch antenna', Elec. Lett., $6^{\text {th }}$ Jan 1994, Vol. 30 , No.1, pp 1-2.

[5] J-S Row, S-H Yeh and K-L Wong.: 'A Wide-band Monopolar Plate-Patch antenna', IEEE Trans Vol. AP-50, No. 9, Sept. 2002, pp 1328-1330.

[6] J-S Row, S-H Yeh, K-L Wong:‘ A Broadband Low-Profile Cylindrical Monopole Antenna', Micro Opt Lett, Vol 29. No. 2, April 2001, pp77-79.

[7] K.L Lau and K.M Luk: ‘A Wide-Band Monopolar Wire-Patch Antenna for Indoor Base Station Applications' IEEE Ant \& Wireless Prop. Lett, Vol.4 2005 pp155-157.

[8] R.Leelaratne and R. Langley: 'Multiband PIFA Vehicle Telematics Antennas', IEEE Trans. Veh. Tech, Vol. 54 No. 2 March 2005 , pp 477-485. 